\title{
Study on the Treatment of Tumor by Immunotherapy with Integrated Traditional Chinese and Western Medicine
}

\author{
Weiyan Qiu Yucheng Huang Zhuanxu Hua
}

Yang Jizhou Chinese Medicine Research Center, Quzhou, Zhejiang, 324000, China

\begin{abstract}
The 2018 Nobel Prize in Medicine was awarded to James Allison, an immunologist at the University of Texas at Austin, and Honjo Ta$\mathrm{suku}^{[1]}$, a professor at Kyoto University in Japan, for their discovery of "immunosuppressive" cancer therapy. The new breakthrough in immunotherapy ${ }^{[2]}$ has greatly promoted people's confidence in defeating cancer. At the same time, traditional Chinese medicine also plays an important role in the field of immunity.
\end{abstract}

\section{Keywords}

immunization; traditional Chinese and Western medicine; tumor

\section{对中西医结合免疫疗法治肿瘤的研究}

装维焰 黄昱程 化转旭

杨继洲中医药研究中心，中国・浙江 锥州 324000

\section{摘 要}

2018 年度诺贝尔医学奖颁给了美国德州大学奥斯汀分校免疫学家詹姆斯・艾利森和日本京都大学教授本庶佑 ${ }^{[1]}$, 以表彰他们 发现了“抑制免疫调节”的癌症疗法。免疫疗法 ${ }^{[2]}$ 的新突破极大的推动了人们战胜癌症的信心, 同时中医药在免疫领域也发 挥着重要的作用。

关键词

免疫; 中西医; 肿瘤

\section{1 引言}

随着中国人口老龄化趋势加剧、以及不良生活方式广泛 存在, 中国癌症发病和死亡率还将不断上升。近十年来每年 增加约 $4 \%$, 调整年龄因素之后, 每年增长约 $2 \%$ 。人口老龄化, 空气环境污染, 不健康饮食, 身体活动不足, 肥胖或超重, 吸烟等等癌症的诱发因素, 除了传统的手术放化疗, 寻求中 西医结合疗法也成为了晚期癌症患者选择的重要手段之一。 对放疗、化疗不能坚持治疗, 对手术、化疗或放疗治疗失效 的病人, 对生理功能减退、多系统疾病共存的老年人及体弱 病人, 属于中医治疗空间, 中医治疗成为当前如何发挥中医 优势治疗肿瘤的重要问题。为了提升晚期肿瘤患者的生存率 和生活质量, 杭州杨继洲研究中心也提出了中西医结合治疗 肿瘤的方法, 采用免疫的机理, 运用中医体质的思想, 以体 质和免疫为出发点, 以人为本来进行辅助治疗。此种疗法的
具体机理如何呢? 主要分三个方面。

\section{2 调整偏颇体质 ${ }^{[3]}$}

体质的平衡意味着对身体五脏六腑的生理功能、精气血 津液的调控, 使之达到一种相对稳定的平衡。

中医体质学创始人王琦提出了九种体质, 分为气虚, 气有, 阴虚, 阳虚, 痰湿, 湿热, 血瘀, 特禀, 平和。而很多人可 以是多种体质类型的结合。体质是中医学很重要的一个概念, 2009 年 4 月中华中医药学会发布的《中医体质分类与判定》 认为, 中医体质是指人体生命过程中, 在先天禀赋及后天获 得基础上所形成的形态结构、生理功能与心理状态方面综合 的、相对稳定的固有特质。

脏腑主要指五脏六腑, 像肝心脾肺肾, 各自发挥自己的 功能, 调整气血津液的运行输布。筋骨的强健, 四肢的灵活, 水液的代谢，思维的敏捷等等都是脏腑各方面协调的结果。 
因此这种特性受到脏腑, 精气血津液等的调控。而脏腑生理 功能否正常发挥、气血津液能否正常运行和推动直接决定着 疾病的各个阶段, 也决定着疾病的发生与否。王琦教授也证 实疾病与体质具有相关性，体质在生化指标、能量代谢的差 异性为体质疾病的易感性及疾病过程的倾向性提供证据。人 体内部以及人体与自然界之间是一个阴阳平衡的整体，一旦 这种平衡因为各种原因被打破, 疾病就随之产生。因此体质 作为内环境的基础, 决定着疾病的发生、发展与转归。癌症 作为一种可控可治的慢性病，晚期癌症患者通过适当治疗可 以控制，获得较好的生活质量进而延长生存时间。

平衡体质运用亳方 ${ }^{[4]}$ 来调补。中药八大剂型丸、散、亳、丹、 酒、露、汤、锭。在这八种剂型里, 亮滋浓缩细淢、药性缓和、 药力持久, 是把配料加水煎謷, 滤去渣滓, 再加进水、冰糖、 蜂蜜等㥿成，是一种具有高级营养滋补和治疗预防综合作用 的成药。

\section{3 改善免疫失调}

肿瘤免疫治疗是通过重新启动并维持肿瘤 - 免疫循环, 恢复机体正常的抗肿瘤免疫反应，从而控制与清除肿瘤的一 种治疗方法。分为非特异性 (活化免疫细胞, 封锁癌细胞将 其杀灭, 对细菌病毒感染引起感冒, 肝炎等抵抗力低下也有效） 和特异性 (找出癌症特定抗原, 体内或体外制造抗体) 疗法, 生物免疫疗法 (依赖于机体自身的力量去杀伤肿瘤细胞), 人体自身免疫疗法 (利用药物, 向肿瘤释放 $\mathrm{T}$ 细胞) 等。人 体免疫失调, 容易生病, 肿瘤也更容易发生, 同样肿瘤患者, 免疫功能也易受到抑制, 二者互为因果。

在中医药领域, 十大仙草之一的灵芝又被誉为 ‘瑞草” , 具有滋补强壮、健脑益肾的作用。其富含的灵芝三萜类（灵 芝酸）是灵芝抑制肿瘤、发挥抗病保健作用的主要成分，灵 芝多糖具有刺激宿主非特异性抗性、免疫特异反应以及抑制 移植肿瘤生理活性的特性。在西医的药理上，中药灵芝同样 发挥着抑瘤防瘤的作用，加上灵芝的保肝，降血脂等作用， 养生灵芝的药用价值非常高。

\section{4 缓解恶病质}

癌症恶病质患者有食欲不振、极度消瘦、衰竭及全身代 谢紊乱、脏器不同程度受损坏等表现的综合征, 中医学将其
归于 “虚劳” 范畴, “虚劳” 发病的病理机制为五脏阴阳气 血虚损。癌症病人中，大于 $80 \%$ 的晚期肿瘤患者中都存在此 种综合征。改善这种综合征，可以选用糖皮质激素和甲地孕

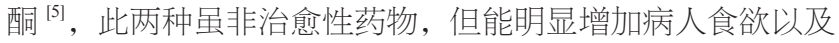
进食量, 并增加体重, 改善营养指标。

《素问・生气通天论》: “故阳气者, 一日而主外, 平旦人气生, 日中而阳气隆, 日西而阳气已需, 气门乃闭。” 人体的阳气与自然界阴阳消长的变化密切相关。阳气在白天 主司于体表。一天之中, 早晨, 阳气开始生发, 中午, 阳 气最为隆盛, 太阳西下时, 阳气渐渐潜藏于里, 汗孔随之 关闭。

从上文可以看出人体阳气一天之中的变化, 而移光定位 针炎疗法就是根据子午流注时间, 太阳光照之时, 阳气最旺 盛而旋转体位而选择针多的一种方法。在临床上, 很多疾病 都和阳气不足有关, 比如癌症、不孕不育、肥胖、内分泌紊乱、 肾病等等。

现代医学化疗、放疗属 “苦寒” “克消” “攻伐” 等范畴, 久之必将损伤脾胃, 损伤人体之阳, 使不足之 “阳” 变得更弱, 助长肿瘤发展。手术摘除肿瘤或者放、化疗之后, 瘤毒虽已 去十之八九，体内痰瘀毒邪并未清除，这也是肿瘤复发难治 的根本原因。病势却为正虚邪恋, 亟需补虚扶正, 除邪务尽, 防死灰复燃, 所以在缓解巩固期, 以中药缓图, 针尒补阳气, 纠正 “化气” 不足, 抑制阴成形太过, 使身体处于阳化气、 阴成形的平衡状态, 肿瘤就失去生长环境。

中医认为腹为阴, 背为阳。根据子午留住时间调整针尒 位置以及其对应的十二条经脉循经取穴, 背对太阳适当光照 以扶阳而施针炎。像胆经上的阳陵泉、胃经上的足三里, 其 他如后溪, 凤池、大椎、神阙、关元、中脘、命门、天枢、 气海等等扶正穴位。移光定位针炎通过培补阳气、正气，有 效提高身体免疫力, 起到抗肿瘤的作用。

总之, 通过平衡体质, 改善免疫功能, 缓解恶病质三位 一体, 并实施培补正气的针食, 对于改善癌症患者症状是有 着明显作用的, 近些年来有大量患者的症状得到改善, 生活 质量也得到相应提高, 除了加强辅助治疗外, 患者能够做到 饮食规律, 起居有节, 不房劳过度也至关重要, 远离那些癌 症诱发的因素。抗癌之路漫漫, 只要我们坚定信心, 积极寻 求和探索新疗法, 癌症在未来终将不再 “谈癌色变”。 
综述性文章
Review

\section{参考文献}

[1] 黄波.2018 年肿瘤免疫研究热点回眸 [J]. 科技导报, 2019,37(1):87-90

[2] 施米丽, 刘晓波. 肿瘤的免疫疗法及其作用机制概述动物医学进 展 2018(32):61-62.

[3] 黄沐, 李爱武, 刘钊汝. 浅谈癌症与中医体质的相关性 [J]. 时珍国
亚洲临床医学杂志・第 02 卷・第 03 期・ 2019 年 08 月 DOI: https://doi.org/10.26549/yzlcyxzz.v2i3.1867

医国药 2007(04):43-45.

[4] 陈捷, 鱼麦侠. 中医亳方调治恶性肿瘤临床应用中国中医药现代 远程教育, 2013(05):13-15.

[5] 赵征. 白新宽甲地孕酮辅助肿瘤化疗的临观察 [J]. 陕西肿瘤医 学, 2001(22):4-5. 